\title{
State bounding estimation for a linear continuous-time singular system with time-varying delay
}

\author{
Jianying $\mathrm{Xiao}^{1 *}$ and Fang $\mathrm{Xu}^{\mathrm{T}}$
}

"Correspondence:

shawion1980@yahoo.com

${ }^{1}$ School of Sciences, Southwest

Petroleum University, Chengdu, China

\section{每 Springer}

\begin{abstract}
This paper investigates the problem of a state bounding estimation for a linear continuous-time singular system with time-varying delay. By employing the maximal Lyapunov-Krasovskii functional and applying the new free-matrix-based integral inequality, some proper conditions are derived in terms of LMIs and a bounding estimation lemma and set are obtained for the studied singular system.
\end{abstract}

Keywords: State bounding estimation; Time delays; Singular system

\section{Introduction}

During the past years, state bounding estimation has been widely applied in control systems with actuator saturation, peak-to-peak gain minimization, and parameter estimation (see [1-5]). A state bounding estimation is meant to get the corresponding state bounding set which is limited by the inside and outside of the initial conditions. State bounding estimation is so important that it has aroused much attention in control research. Meanwhile, there have been several kinds of correlative applications of a state bounding estimation, such as reachable set estimation and design actuator saturation for control systems (see [1-53]).

Specially, by applying the S-procedure, an ellipsoid reachable set bounding was derived for linear systems without time delays in [19]. However, time delays cannot always be avoided in practice and they often cause the system's instability and poor performance. Recently, many researchers have studied many kinds of dynamic systems with time delays (see [3, 6-25]). Thus, some researchers naturally have devoted efforts to investigating the corresponding state bounding estimation for the dynamic systems with time delays. In [7], a delay-dependent criterion for an ellipsoid reachable bounding set was derived by Fridman and Shaked, applying a Lyapunov-Krasovskii functional. Later, in [11], a better ellipsoid reachable bounding set was proposed by Kim using a Lyapunov-Krasovskii functional with just one nonconvex scalar. Some new criteria for reachable bounding sets were established by employing the maximal Lyapunov-Krasovskii functional in [14].

On the other hand, singular systems have been more intensively studied than state-space ones because they can present a better description of the behavior for some systems. There have been many singular systems in lots of practical systems, such as circuit systems, aircraft modeling, chemical processes and economic systems. Leaving alone their practical

(c) The Author(s) 2019. This article is distributed under the terms of the Creative Commons Attribution 4.0 International License (http://creativecommons.org/licenses/by/4.0/), which permits unrestricted use, distribution, and reproduction in any medium, provided you give appropriate credit to the original author(s) and the source, provide a link to the Creative Commons license, and indicate if changes were made. 
performance, singular systems are worth investigating for their theoretical importance and have drawn many researchers' attention in recent years because their basis is different from state-space systems. However, many researchers have successfully extended the basic theory of state-space systems to the singular systems. Recently, there have been several contributions on the state bounding estimation for nonlinear singular systems by applying different methods (see [11-19]). Particulary, Feng and Lam in [17] obtained the reachable set estimation for singular systems with time delays by using a Lyapunov-Krasovskii functional but not the maximal Lyapunov-Krasovskii functional.

In this paper, we extend the state bounding estimation to a singular system with timevarying delay. By employing the maximal Lyapunov-Krasovskii functional and applying the new free-matrix-based integral inequality, some proper conditions are derived in terms of LMIs and a new bounding estimation lemma and set are obtained for the studied singular system.

Notations: Throughout this paper, $R^{n}$ denotes $n$-dimensional Euclidean space and $R^{m \times n}$ is the set of $m \times n$ real matrices. [., , ] represents an interval. The superscripts ' -1 ' and ' $T$ ' stand for the inverse and transpose of a matrix, respectively. $\|\cdot\|$ refers to the Euclidean vector norm. $*$ denotes the symmetric block in a symmetric matrix. $\operatorname{Sym}(P)$ is defined as $P+P^{T}$. For a real number $\epsilon$, use the notation $\epsilon^{+}=\max \{\epsilon, 0\}$, which means $\epsilon^{+}=\left\{\begin{array}{l}\epsilon, \epsilon>0, \\ 0, \epsilon \leq 0\end{array}\right.$ Matrix dimensions, if not explicitly stated, are assumed to be compatible for algebraic operations.

\section{Problem statement and preliminaries}

Consider the following linear continuous-time singular system with time-varying delay:

$$
\begin{aligned}
& E \dot{x}(t)=A x(t)+D x(t-d(t))+B u(t), \\
& x(t)=\phi(t), \quad t \in\left[-d_{M}, 0\right],
\end{aligned}
$$

where $x(t) \in R^{n}$ is the state vector; the matrices $E, A, D$ and $B$ are constant matrices with appropriate dimensions and $\operatorname{rank}(E)=n_{1} ; d(t)$ is the time-varying delay satisfying $0 \leq d_{m} \leq d(t) \leq d_{M}, \dot{d}(t)<d, d>0 ; u(t) \in R^{m}$ stands for a disturbance which satisfies $u^{T}(t) u(t) \leq u^{2} \leq\left\|x_{t}\right\|^{2}$, where $u$ is a real constant; $\phi(t)$ is the initial condition and continuously differentiable on $\left[-d_{M}, 0\right]$. We denote $\|\phi\|=\max _{t \in\left[-d_{M}, 0\right]}\|\phi\|$.

Remark 1 The initial condition for the studied system in [17] must be zero. However, the initial condition in this paper either may be equal to zero or not equal to zero. It is obvious that our discussed system is more general than the one in [17].

To discuss the state bounding problem for a linear continuous-time singular system with time-varying delay, the next definitions are necessary.

Definition 2.1 If the singular system (1) is satisfied with one of the next conditions, respectively, the system (1) is said to be regular, impulse free, stable and admissible:

(1) The singular system in (1) is said to be regular if the matrix pair $(E, A)$ is regular.

(2) The singular system in (1) is said to be impulse free if the matrix pair $(E, A)$ is impulse free. 
(3) The singular system in (1) is said to be stable, if for any $\delta>0$, there exists a scalar $\varepsilon(\delta)>0$, such that, for any compatible initial condition $x_{0}$ satisfying $\left\|x_{0}\right\| \leq \varepsilon(\delta)$, the solution $x(t)$ of (1) satisfies $\|x(t)\| \leq \delta$ for $t \geq 0$; furthermore, $x(t) \rightarrow 0$, when $t \rightarrow \infty$.

(4) The singular system in (1) is said to be admissible if it is regular, impulse free and stable.

Definition 2.2 For a given $\alpha>0$, system (1) with $u(t)=0(t \geq 0)$ is said to be $\alpha$ exponentially stable if there exists a positive constant $\rho$ such that every solution $x(t, \phi)$ of (1) satisfies the following inequality:

$$
\|x(t, \phi)\| \leq \rho\|\phi\| e^{-\alpha t}, \quad \forall t \geq 0 .
$$

For $\gamma>0$, let the ball $\mathcal{B}(\gamma)$ be defined by $\mathcal{B}(\gamma)=\left\{x \in R^{n}:\|x\| \leq \gamma\right\}$. By adopting the concept of ball convergence in [16], we have the following definition.

Definition 2.3 For a given $\gamma>0$, the system (1) is said to be globally exponentially convergent within the ball $\mathcal{B}(\gamma)$ if there exist a constant $\alpha>0$ and a non-decreasing function $K(\cdot)$ such that the following inequality holds:

$$
\|x(K, \phi)\| \leq \gamma+K(\|\phi\|) e^{-\alpha t}, \quad \forall t \geq 0 .
$$

The main objective of this paper is to obtain delay-dependent conditions for the state bounding problem of singular system (1). First, the conditions are investigated for the existence of two balls, namely, $\mathcal{B}\left(\gamma_{1}\right)$ and $\mathcal{B}\left(\gamma_{2}\right)$ with the radii $\gamma_{1}, \gamma_{2}$ explicitly dependent on the upper bound $u^{2}$ of the disturbance such that: (i) for all initial conditions in $\mathcal{B}\left(\gamma_{1}\right)$, the corresponding state trajectories of the systems are bounded inside the ball $\mathcal{B}\left(\gamma_{2}\right)$ all the time, and (ii) for all the initial conditions that are outside $\mathcal{B}\left(\gamma_{1}\right)$, the corresponding state trajectories of the systems converge exponentially (with a convergence rate $\alpha$ ) within $\mathcal{B}\left(\gamma_{2}\right)$. Then the conditions are derived for the reachable set bounding of (1) with zero initial condition and the $\alpha$-exponential stability of (1) without any disturbance.

In the following, some necessary lemmas are introduced.

Lemma 2.1 ([18]) Let $x$ be a differentiable function: $[a, b] \rightarrow R^{n}$. For symmetric matrices $Q \in R^{n \times n}, Y_{1} \in R^{3 n \times 3 n}, Y_{3} \in R^{3 n \times 3 n}$ and any matrices $Y_{2} \in R^{3 n \times 3 n}, M_{1} \in R^{3 n \times n}, M_{2} \in R^{3 n \times n}$ satisfying

$$
\Psi=\left[\begin{array}{ccc}
Y_{1} & Y_{2} & M_{1} \\
* & Y_{3} & M_{2} \\
* & * & Q
\end{array}\right] \geq 0,
$$

the following integral inequality holds:

$$
-\int_{a}^{b} \dot{x}^{T}(s) Q \dot{x}(s) d s \leq \varpi^{T} \Omega \varpi,
$$

where

$$
\varpi=\left[x^{T}(a), x^{T}(b), \frac{1}{b-a} \int_{a}^{b} \dot{x}^{T}(s) d s\right]^{T},
$$




$$
\begin{aligned}
& \Omega=\left[(b-a)\left(Y_{1}+\frac{1}{3} Y_{3}\right)\right]+\operatorname{Sym}\left\{M_{1} \Pi_{1}+M_{2} \Pi_{2}\right\}, \\
& \Pi_{1}=e_{1}-e_{2}, \quad \Pi_{2}=2 e_{3}-e_{1}-e_{2}, \\
& e_{1}=[I, 0,0], \quad e_{2}=[0, I, 0], \quad e_{3}=[0,0, I] .
\end{aligned}
$$

Lemma 2.2 ([17]) Let $0 \leq \tau_{m}<\tau_{M}, 0<\lambda<1, Q \geq 0, x(t)$ be a continuous vector-valued function on $\left[\tau_{m}, \tau_{M}\right]$.If $\|x(t)\| \leq \lambda\|x(t-\tau(t))\|+Q, t \geq 0$, then $\|x(t)\| \leq \sup _{-\tau_{M} \leq t \leq 0}\|x\|+\frac{Q}{1-\lambda}$.

Lemma 2.3 ([17]) If system (1) is regular and impulse free, there exist two nonsingular matrices $M$ and $N$ such that

$$
M E N=\left[\begin{array}{cc}
I_{n_{1}} & 0 \\
0 & 0
\end{array}\right], \quad M A N=\left[\begin{array}{cc}
\hat{A}_{1} & 0 \\
0 & I
\end{array}\right] .
$$

Let

$$
\hat{x}(t)=N^{-1} x(t)=\left[\begin{array}{l}
\hat{x}_{1}(t) \\
\hat{x}_{2}(t)
\end{array}\right],
$$

where $\hat{x}_{1}(t) \in R^{n_{1}}$ and $\hat{x}_{2}(t) \in R^{n-n_{1}}$. Denote

$$
M A_{\tau} N=\left[\begin{array}{ll}
\hat{D}_{11} & \hat{D}_{12} \\
\hat{D}_{21} & \hat{D}_{22}
\end{array}\right], \quad M B=\left[\begin{array}{l}
\hat{B}_{1} \\
\hat{B}_{2}
\end{array}\right] .
$$

Then system (1) is a restricted system, equivalent to the following one:

$$
\begin{aligned}
& \dot{\hat{x}}_{1}(t)=\hat{A}_{1} \hat{x}_{1}(t)+\hat{D}_{11} \hat{x}_{1}(t-\tau(t))+\hat{D}_{12} \hat{x}_{2}(t-\tau(t))+\hat{B}_{1} u(t), \\
& 0=\hat{x}_{2}(t)+\hat{D}_{21} \hat{x}_{1}(t-\tau(t))+\hat{D}_{22} \hat{x}_{2}(t-\tau(t))+\hat{B}_{2} u(t) .
\end{aligned}
$$

Lemma 2.4 Assume that there exist a functional $V\left(x_{t}\right)$ and positive scalars $\lambda_{1}, \lambda_{2}, \alpha, \beta \in$ $(0,1)$ such that

$$
\begin{aligned}
& \text { (1) } \lambda_{1}\|x(t)\|^{2} \leq V\left(x_{t}\right) \leq \lambda_{2}\left\|x_{t}\right\|^{2}, \quad t \geq 0, \\
& \text { (2) } \dot{V}\left(x_{t}\right)+\alpha V\left(x_{t}\right) \leq \beta u^{T}(t) u(t),
\end{aligned}
$$

where $x_{t}$ represents the state trajectory $\left\{x(t+\theta): \theta \in C\left[-d_{M}, 0\right]\right\}$. Then every solution $x(t, \phi)$ of (1) satisfies

$$
\|x(t, \phi)\|^{2} \leq \frac{\beta u^{2}}{\alpha \lambda_{1}}+\left(\frac{\lambda_{2}}{\lambda_{1}}\|\phi\|^{2}-\frac{\beta u^{2}}{\alpha \lambda_{1}}\right)^{+} e^{-\alpha t}, \quad \forall t \geq 0 .
$$

Proof Notice

$$
\dot{V}\left(x_{t}\right)+\alpha V\left(x_{t}\right) \leq \beta u^{T}(t) u(t) .
$$

By multiplying both sides of the inequality in (7) with $e^{\alpha t}$, we have

$$
e^{\alpha t} \dot{V}\left(x_{t}\right)+\alpha e^{\alpha t} V\left(x_{t}\right)=\frac{d}{d t}\left(e^{\alpha t} V\left(x_{t}\right)\right) \leq \beta e^{\alpha t} u^{T}(t) u(t) .
$$


Then, by performing the integral of (10) from 0 to $T>0$, it is not difficult to obtain

$$
e^{\alpha t} V\left(x_{T}\right)-V\left(x_{0}\right)=\int_{0}^{T} \beta e^{\alpha t} u^{T}(t) u(t) d t \leq \int_{0}^{T} \beta e^{\alpha t} u^{2} d t=\frac{\beta}{\alpha} u^{2}\left(e^{\alpha T}-1\right) .
$$

By simple computation, it is easy to get

$$
V\left(x_{T}\right) \leq e^{-\alpha t} V\left(x_{0}\right) \frac{\beta}{\alpha} u^{2}\left(e^{\alpha T}-1\right) e^{-\alpha T}=\frac{\beta}{\alpha} u^{2}+\left[V\left(x_{0}\right)-\frac{\beta}{\alpha} u^{2}\right] e^{-\alpha T} .
$$

Finally, for all $t \geq 0$, replacing $T$ with $t$ in (12), we get

$$
V\left(x_{t}\right) \leq \frac{\beta}{\alpha} u^{2}+\left[V\left(x_{0}\right)-\frac{\beta}{\alpha} u^{2}\right] e^{-\alpha t} .
$$

Taking (6) into account, we derive

$$
\|x(t, \phi)\|^{2} \leq \frac{\beta u^{2}}{\alpha \lambda_{1}}+\left(\frac{\lambda_{2}}{\lambda_{1}}\|\phi\|^{2}-\frac{\beta u^{2}}{\alpha \lambda_{1}}\right)^{+} e^{-\alpha t} .
$$

The proof is thus completed.

Remark 2 Lemma 2.4 in this paper provides a basic tool for the problem of state bounding not only containing the state convergence within a ball but also including the reachable set bounding for a continuous-time singular system with bounded disturbance input. It is obvious that Lemma 2.4 in this paper can be regarded as an expansion of Lemma 3 in [17]. Particularly, taking $\beta=\frac{\alpha}{u^{2}}$ and $V\left(x_{0}\right)$, it is easy to see that Lemma 2.4 is reduced to Lemma 3 in [17], which was proved to be more useful for the reachable set estimation of singular systems with bounded disturbances. Note that Lemma 2.4 in this paper can also be applied to the case where there is no disturbance in system (1). In this case, the studied problem is reduced to the $\alpha$-exponential stability analysis for a singular system with interval time-varying delay.

\section{Main results}

According to Lemma 2.3, we consider the following system's state bounding estimation:

$$
\begin{aligned}
& \dot{\hat{x}}_{1}(t)=\hat{A}_{1} \hat{x}_{1}(t)+\hat{D}_{11} \hat{x}_{1}(t-\tau(t))+\hat{D}_{12} \hat{x}_{2}(t-\tau(t))+\hat{B}_{1} u(t), \\
& 0=\hat{x}_{2}(t)+\hat{D}_{21} \hat{x}_{1}(t-\tau(t))+\hat{D}_{22} \hat{x}_{2}(t-\tau(t))+\hat{B}_{2} u(t) .
\end{aligned}
$$

Assumption 1 The matrix pair $(E, D)$ is regular and $\left\|\hat{D}_{22}\right\|<1$.

Theorem 3.1 Under Assumption 1, the singular system (1) is bounded by the ellipsoid

$$
\begin{aligned}
& \mathcal{B}(\varepsilon)=\left\{x \in R^{n} \mid x^{T} \hat{\hat{P}}_{j} x \leq \frac{\beta u^{2}}{\alpha}\right\} \\
& \left(\hat{P}_{j}=\left(S^{-T} P_{j} S^{-1}\right)_{n_{1} \times n_{1}}, \hat{\hat{P}}_{j}=T^{-T}\left[\begin{array}{cc}
\eta \hat{P}_{j} & 0 \\
0 & \frac{(1-\eta) \beta u^{2}}{\epsilon_{2}^{2} \alpha}
\end{array}\right] T^{-1}\right)
\end{aligned}
$$


if there exist positive $R^{n \times n}$ matrices $P_{j}, Q_{1}, Q_{2}, Q_{3}, X_{1}, X_{2}, G_{1}, G_{2}, G_{3}, G_{4}, G_{5}, G_{6}, G_{7}, G_{8}$, any $R^{3 n \times 3 n}$ symmetric matrices

$$
\begin{array}{rlrl}
Y_{1} & =\left[\begin{array}{lll}
Y_{11} & Y_{12} & Y_{13} \\
Y_{21} & Y_{22} & Y_{23} \\
Y_{31} & Y_{32} & Y_{33}
\end{array}\right], & Y_{3} & =\left[\begin{array}{lll}
\bar{Y}_{11} & \bar{Y}_{12} & \bar{Y}_{13} \\
\bar{Y}_{21} & \bar{Y}_{22} & \bar{Y}_{23} \\
\bar{Y}_{31} & \bar{Y}_{32} & \bar{Y}_{33}
\end{array}\right], \\
Z_{1} & =\left[\begin{array}{lll}
Z_{11} & Z_{12} & Z_{13} \\
Z_{21} & Z_{22} & Z_{23} \\
Z_{31} & Z_{32} & Z_{33}
\end{array}\right], & Z_{3}=\left[\begin{array}{lll}
\bar{Z}_{11} & \bar{Z}_{12} & \bar{Z}_{13} \\
\bar{Z}_{21} & \bar{Z}_{22} & \bar{Z}_{23} \\
\bar{Z}_{31} & \bar{Z}_{32} & \bar{Z}_{33}
\end{array}\right],
\end{array}
$$

any $R^{3 n \times 3 n}$ matrices $Y_{2}, Z_{2}$, and any $R^{3 n \times n}$ matrices

$$
N_{1}=\left[\begin{array}{l}
N_{11} \\
N_{12} \\
N_{13}
\end{array}\right], \quad N_{2}=\left[\begin{array}{l}
N_{21} \\
N_{22} \\
N_{23}
\end{array}\right], \quad M_{1}=\left[\begin{array}{l}
M_{11} \\
M_{12} \\
M_{13}
\end{array}\right], \quad M_{2}=\left[\begin{array}{l}
M_{21} \\
M_{22} \\
M_{23},
\end{array}\right]
$$

such that the following matrix inequalities hold:

$$
\begin{aligned}
& \Omega=\left(\Omega_{i j}\right)_{8 \times 8} \quad(i, j=1,2, \ldots, 8)<0, \\
& \Omega_{1}=\left[\begin{array}{ccc}
Y_{1} & Y_{2} & M_{1} \\
* & Y_{3} & M_{2} \\
* & * & E^{T} X_{1} E
\end{array}\right] \geq 0, \\
& \Omega_{2}=\left[\begin{array}{ccc}
Z_{1} & Z_{2} & N_{1} \\
* & Z_{3} & N_{2} \\
* & * & E^{T} X_{2} E
\end{array}\right] \geq 0,
\end{aligned}
$$

where

$$
\begin{aligned}
\Omega_{i j}= & \Omega_{j i} \quad(i, j=1,2, \ldots, 8), \\
\Omega_{11}= & Q_{1}+Q_{2}+Q_{3}+\alpha E^{T} P_{j} E-e^{-\alpha d_{m}} E^{T} X_{2} E+\operatorname{sym}\left(G_{1}^{T} A\right), \\
\Omega_{12}= & -e^{-\alpha d_{m}} E^{T} X_{2} E+A^{T} G_{2}, \\
\Omega_{13}= & G_{1}^{T} D+A^{T} G_{3}, \\
\Omega_{14}= & G_{4}^{T} A, \\
\Omega_{15}= & A^{T} G_{5}, \\
\Omega_{16}= & A^{T} G_{6}, \\
\Omega_{17}= & \left(P_{j} E+E_{0} U\right)^{T}-G_{1}^{T}+A^{T} G_{7}, \\
\Omega_{18}= & G_{1}^{T} B, \\
\Omega_{22}= & -e^{-\alpha d_{m}} E^{T} X_{2} E-e^{-\alpha d_{m}} Q_{1}+\left(d_{M}-d_{m}\right)^{2} e^{-\alpha d_{M}}\left(Y_{11}+\frac{\bar{Y}_{11}}{3}\right) \\
& +\left(d_{M}-d_{m}\right)^{2} e^{-\alpha d_{M}}\left(M_{11}+M_{21}\right),
\end{aligned}
$$




$$
\begin{aligned}
& \Omega_{23}=G_{2}^{T} D+\left(d_{M}-d_{m}\right)^{2} e^{-\alpha d_{M}}\left(Y_{12}+\frac{\bar{Y}_{12}}{3}\right)+\frac{\left(d_{M}-d_{m}\right)^{2} e^{-\alpha d_{M}}}{2}\left(M_{12}-M_{11}\right) \\
& -\frac{\left(d_{M}-d_{m}\right)^{2} e^{-\alpha d_{M}}}{2}\left(M_{21}+M_{22}\right) \\
& \Omega_{25}=\left(d_{M}-d_{m}\right)^{2} e^{-\alpha d_{M}}\left(Y_{13}+\frac{\bar{Y}_{13}}{3}\right)+\frac{\left(d_{M}-d_{m}\right)^{2} e^{-\alpha d_{M}}}{2}\left(2 M_{13}+M_{21}-M_{23}\right), \\
& \Omega_{27}=-G_{2}^{T}, \\
& \Omega_{28}=G_{2}^{T} B, \\
& \Omega_{33}=-(1-\mu) e^{-\alpha d_{m}} Q_{3}+\operatorname{sym}\left(G_{3}^{T} D\right)+\left(d_{M}-d_{m}\right)^{2} e^{-\alpha d_{M}}\left(Y_{22}+\frac{\bar{Y}_{22}}{3}+Z_{11}+\frac{\bar{Z}_{11}}{3}\right) \\
& +\frac{\left(d_{M}-d_{m}\right)^{2} e^{-\alpha d_{M}}}{2}\left(2 M_{12}-2 M_{22}+2 N_{12}-2 N_{21}\right), \\
& \Omega_{34}=D^{T} G_{4}+\left(d_{M}-d_{m}\right)^{2} e^{-\alpha d_{M}}\left(Z_{12}+\frac{\bar{Z}_{12}}{3}\right) \\
& +\frac{\left(d_{M}-d_{m}\right)^{2} e^{-\alpha d_{M}}}{2}\left(N_{12}-N_{11}-N_{22}-N_{21}\right) \text {, } \\
& \Omega_{35}=D^{T} G_{5}+\left(d_{M}-d_{m}\right)^{2} e^{-\alpha d_{M}}\left(Y_{23}+\frac{\bar{Y}_{23}}{3}\right)+\frac{\left(d_{M}-d_{m}\right)^{2} e^{-\alpha d_{M}}}{2} M_{13}, \\
& \Omega_{36}=D^{T} G_{6}+\left(d_{M}-d_{m}\right)^{2} e^{-\alpha d_{M}}\left(Z_{13}+\frac{\bar{Z}_{13}}{3}\right)+\frac{\left(d_{M}-d_{m}\right)^{2} e^{-\alpha d_{M}}}{2}\left(N_{13}+2 N_{21}-N_{23}\right), \\
& \Omega_{37}=D^{T} G_{7}-G_{3}^{T}, \\
& \Omega_{38}=G_{3}^{T} B, \\
& \Omega_{44}=-e^{-\alpha d_{M}} Q_{2}+\left(d_{M}-d_{m}\right)^{2} e^{-\alpha d_{M}}\left(Z_{22}+\frac{\bar{Z}_{22}}{3}\right)-\frac{\left(d_{M}-d_{m}\right)^{2} e^{-\alpha d_{M}}}{2}\left(N_{12}+2 N_{22}\right), \\
& \Omega_{46}=\left(d_{M}-d_{m}\right)^{2} e^{-\alpha d_{M}}\left(Z_{23}+\frac{\bar{Z}_{23}}{3}\right)+\frac{\left(d_{M}-d_{m}\right)^{2} e^{-\alpha d_{M}}}{2}\left(-N_{13}+2 N_{22}-N_{23}\right), \\
& \Omega_{48}=-G_{4}^{T}+G_{4}^{T} B, \\
& \Omega_{55}=\left(d_{M}-d_{m}\right)^{2} e^{-\alpha d_{M}}\left(Y_{33}+\frac{\bar{Y}_{33}}{3}\right)+2\left(d_{M}-d_{m}\right)^{2} e^{-\alpha d_{M}} M_{23}, \\
& \Omega_{57}=-G_{5}^{T}, \\
& \Omega_{58}=G_{5}^{T} B, \\
& \Omega_{66}=\left(d_{M}-d_{m}\right)^{2} e^{-\alpha d_{M}}\left(Z_{33}+\frac{\bar{Z}_{33}}{3}\right)+2\left(d_{M}-d_{m}\right)^{2} e^{-\alpha d_{M}} N_{23}, \\
& \Omega_{67}=-G_{6}^{T}, \\
& \Omega_{68}=G_{6}^{T} B, \\
& \Omega_{77}=d_{m}^{2} X_{2}+2\left(d_{M}-d_{m}\right)^{2} X_{1}-\operatorname{sym}\left(G_{7}\right), \\
& \Omega_{78}=G_{7}^{T} B, \\
& \Omega_{88}=-\beta I+\operatorname{sym}\left(G_{8}^{T} B\right) .
\end{aligned}
$$


Proof Given a family of matrices $P_{j}>0, Q_{1}>0, Q_{2}>0, Q_{3}>0, X_{1}>0, X_{2}>0$, the pointwise maximum Lyapunov-Krasovskii functional is defined as

$$
\begin{aligned}
V_{\max } & =\max \left\{V_{1, j}(x)+V_{2}\left(x_{t}\right)+V_{3}\left(x_{t}\right)+V_{4}\left(x_{t}\right)\right\}=\max \left\{V_{j}(x)\right\} \\
& =V_{1, \max }(x)+V_{2}\left(x_{t}\right)+V_{3}\left(x_{t}\right)+V_{4}\left(x_{t}\right), \quad j=1,2, \ldots, N,
\end{aligned}
$$

where

$$
\begin{aligned}
V_{1, j}(x)= & x^{T}(t) E^{T} P_{j} E x(t), \\
V_{1, \max }(x) & =\max V_{1, j}(x), \\
V_{2}\left(x_{t}\right)= & \int_{t-d_{m}}^{t} e^{\alpha \theta-t} x^{T}(\theta) Q_{1} x(\theta) d \theta \\
& +\int_{t-d_{M}}^{t} e^{\alpha \theta-t} x^{T}(\theta) Q_{2} x(\theta) d \theta+\int_{t-d(t)}^{t} e^{\alpha \theta-t} x^{T}(\theta) Q_{3} x(\theta) d s, \\
V_{3}\left(x_{t}\right)= & \left(d_{M}-d_{m}\right) \int_{-d_{M}}^{-d_{m}} \int_{t+s}^{t} e^{\alpha \theta-t} \dot{x}^{T}(\theta) E^{T} X_{1} E \dot{x}(\theta) d s d \theta, \\
V_{4}\left(x_{t}\right)= & d_{m} \int_{-d_{m}}^{0} \int_{t+s}^{t} e^{\alpha \theta-t} \dot{x}^{T}(\theta) E^{T} X_{2} E \dot{x}(\theta) d s d \theta .
\end{aligned}
$$

From Lemma 2.4, we have

$$
\lambda_{1}\|x(t)\|^{2} \leq V_{\max } \leq \lambda_{2}\left\|x_{t}\right\|^{2}, \quad t \geq 0
$$

where

$$
\begin{aligned}
\lambda_{1}= & \min _{1 \leq j \leq N} \lambda\left(E^{T} P_{j} E\right), \\
\lambda_{2}= & \max _{1 \leq j \leq N} \lambda\left(E^{T} P_{j} E\right)+\max _{1 \leq j \leq N} \lambda\left(Q_{1}\right) d_{m}+\max _{1 \leq j \leq N} \lambda\left(Q_{2}\right) d_{M}+\max _{1 \leq j \leq N} \lambda\left(Q_{3}\right) d_{M} \\
& +\max _{1 \leq j \leq N} \lambda\left(E^{T} X_{1} E\right)\left(d_{M}-d_{m}\right)^{3}+\max _{1 \leq j \leq N} \lambda\left(E^{T} X_{2} E\right) d_{m}^{2} .
\end{aligned}
$$

In order to better express the idea of our proof, define a set $M_{\max }(x):=\{j \in\{1,2, \ldots, K\}$ : $\left.V_{j}(x)=V_{\max }(x)\right\}$ for any $x \neq 0$. Therefore, $V_{j}(x)<V_{\max }(x)$, if $j \neq M_{\max }(x)$. Without loss of generality, we assume that the first $n$ ellipsoids intersect at $x$. In that case, $M_{\max }=$ $\{1,2, \ldots, n\}$. Thus one obtains for all $k \in\{1,2, \ldots, K\}, x^{T}\left(P_{k}-P_{j}\right) x \leq 0, \forall j \leq n$.

Let $\eta_{j}=\left\{x \in R^{n}: V_{j}(x) \geq V_{k}(x), \forall k \neq j\right\}$. Observe that $x$ is differentiable if $x \in \eta_{j} \backslash V_{k \neq j} \eta_{k}$, while $x$ is nondifferentiable if $x \in \bigcap_{j=1}^{n} \eta_{j} \backslash \bigcup_{j=m+1}^{K} \eta_{j}$.

Since the derivative of a convex function at a differential point can be regarded as a special case of a directional derivative for the same convex function at a nondifferentiable point, we combine these two situations and only illustrate the proof for the situation of a nondifferentiable point in the following discussion. Therefore,

$$
\begin{aligned}
& V_{\max }=x^{T}(t) E^{T} P_{j} E x(t)+V_{2}\left(x_{t}\right)+V_{3}\left(x_{t}\right)+V_{4}\left(x_{t}\right), \quad j \in M_{\max }(x), \\
& \partial V_{1, \max }(x)=C o\left\{\partial P_{j} x\right\}, \quad j \in M_{\max }(x) .
\end{aligned}
$$


Calculating the derivative of $V_{\max }$, we have

$$
\begin{aligned}
& \nabla_{\dot{x}} V_{1, \max }(x)=2 \dot{x}^{T}(t) E^{T}\left(P_{j} E+E_{0} u\right) x(t) \\
& =2(E \dot{x}(t))^{T} P_{j} E x(t)+2(E \dot{x}(t))^{T} P_{j} E_{0} u x(t), \\
& \dot{V}_{2}\left(x_{t}\right)=-\alpha V_{2}+x^{T}(t)\left(Q_{1}+Q_{2}+Q_{3}\right) x(t)-e^{-\alpha d_{m}} x^{T}\left(t-d_{m}\right) Q_{1} x\left(t-d_{m}\right) \\
& -e^{-\alpha d_{M}} x^{T}\left(t-d_{M}\right) Q_{2} x\left(t-d_{M}\right) \\
& -(1-\dot{\tau}(t)) e^{-\alpha d(t)} x^{T}(t-d(t)) Q_{3} x(t-d(t)) \\
& \leq-\alpha V_{2}+x^{T}(t)\left(Q_{1}+Q_{2}+Q_{3}\right) x(t)-e^{-\alpha d_{m}} x^{T}\left(t-d_{m}\right) Q_{1} x\left(t-d_{m}\right) \\
& -e^{-\alpha d_{M}} x^{T}\left(t-d_{M}\right) Q_{2} x\left(t-d_{M}\right) \\
& -(1-\mu) e^{-\alpha d(t)} x^{T}(t-d(t)) Q_{3} x(t-d(t)), \\
& \dot{V}_{3}\left(x_{t}\right)=-\alpha V_{3}+\left(d_{M}-d_{m}\right)^{2} \dot{x}^{T}(t) E^{T} X_{1} E \dot{x}(t) \\
& -\left(d_{M}-d_{m}\right) \int_{t-d_{M}}^{t-d_{m}} e^{\alpha(\theta-t)} \dot{x}^{T}(\theta) E^{T} X_{1} E \dot{x}(\theta) d \theta \\
& \leq-\alpha V_{3}+\left(d_{M}-d_{m}\right)^{2} \dot{x}^{T}(t) E^{T} X_{1} E \dot{x}(t) \\
& -\left(d_{M}-d_{m}\right) e^{-\alpha d_{M}} \int_{t-d_{M}}^{t-d_{m}} \dot{x}^{T}(\theta) E^{T} X_{1} E \dot{x}(\theta) d \theta, \\
& \dot{V}_{4}\left(x_{t}\right)=-\alpha V_{4}+d_{m}^{2} \dot{x}^{T}(t) E^{T} X_{2} E \dot{x}(t)-d_{m} \int_{t-d_{m}}^{t} e^{\alpha(\theta-t)} \dot{x}^{T}(\theta) E^{T} X_{2} E \dot{x}(\theta) d \theta \\
& \leq-\alpha V_{4}+d_{m}^{2} \dot{x}^{T}(t) E^{T} X_{2} E \dot{x}(t)-d_{m} e^{-\alpha d_{m}} \int_{t-d_{m}}^{t} \dot{x}^{T}(\theta) E^{T} X_{2} E \dot{x}(\theta) d \theta .
\end{aligned}
$$

By employing Lemma 2.1, there exist matrices $Y_{1}, Y_{2}, Y_{3}, Z_{1}, Z_{2}, Z_{3} \in R^{3 n \times 3 n}$, and any matrices $N_{1}, N_{2}, M_{1}, M_{2} \in R^{3 n \times n}$ satisfying

$$
\Omega_{1}=\left[\begin{array}{ccc}
Y_{1} & Y_{2} & M_{1} \\
* & Y_{3} & M_{2} \\
* & * & E^{T} X_{1} E
\end{array}\right] \geq 0, \quad \Omega_{2}=\left[\begin{array}{ccc}
Z_{1} & Z_{2} & N_{1} \\
* & Z_{3} & N_{2} \\
* & * & E^{T} X_{2} E
\end{array}\right] \geq 0
$$

such that $d_{m}<d(t)<d_{M}$. We have

$$
\begin{aligned}
\dot{V}_{3}\left(x_{t}\right) \leq & -\alpha V_{3}+\left(d_{M}-d_{m}\right)^{2} \dot{x}^{T}(t) E^{T} X_{1} E \dot{x}(t) \\
& -\left(d_{M}-d_{m}\right) e^{-\alpha d_{M}} \int_{t-d_{M}}^{t-d(t)} \dot{x}^{T}(\theta) E^{T} X_{1} E \dot{x}(\theta) d \theta \\
& -\left(d_{M}-d_{m}\right) e^{-\alpha d_{M}} \int_{t-d(t)}^{t-d_{m}} \dot{x}^{T}(\theta) E^{T} X_{1} E \dot{x}(\theta) d \theta \\
\leq & -\alpha V_{3}+\left(d_{M}-d_{m}\right)^{2} \dot{x}^{T}(t) E^{T} X_{1} E \dot{x}(t) \\
& +\left(d_{M}-d_{m}\right) e^{-\alpha d_{M}}\left[x^{T}\left(t-d_{m}\right), x^{T}(t-d(t)), \frac{1}{d(t)-d_{m}} \int_{t-d(t)}^{t-d_{m}} x^{T}(\theta) d \theta\right] \\
& \times\left[\left(d(t)-d_{m}\right)\left(Y_{1}+\frac{1}{3} Y_{3}\right)\right]
\end{aligned}
$$


Ciao and Xu Advances in Difference Equations

(2019) 2019:120

Page 10 of 17

$$
\begin{aligned}
& \times\left[x^{T}\left(t-d_{m}\right), x^{T}(t-d(t)), \frac{1}{d(t)-d_{m}} \int_{t-d(t)}^{t-d_{m}} x^{T}(\theta) d \theta\right]^{T} \\
& +\left(d_{M}-d_{m}\right) e^{-\alpha d_{M}}\left[x^{T}\left(t-d_{m}\right), x^{T}(t-d(t)), \frac{1}{d(t)-d_{m}} \int_{t-d(t)}^{t-d_{m}} x^{T}(\theta) d \theta\right] \\
& \times \operatorname{Sym}\left(M_{1}\left(x(t-d(t))-x\left(t-d_{m}\right)\right)\right) \\
& +\left(d_{M}-d_{m}\right) e^{-\alpha d_{M}}\left[x^{T}\left(t-d_{m}\right), x^{T}(t-d(t)), \frac{1}{d(t)-d_{m}} \int_{t-d(t)}^{t-d_{m}} x^{T}(\theta) d \theta\right] \\
& \times \operatorname{Sym}\left(M_{2}\left(\frac{2}{d(t)-d_{m}} \int_{t-d(t)}^{t-d_{m}} x^{T}(\theta) d \theta-x(t-d(t))-x\left(t-d_{m}\right)\right)\right) \\
& +\left(d_{M}-d_{m}\right) e^{-\alpha d_{M}}\left[x^{T}(t-d(t)), x^{T}\left(t-d_{M}\right), \frac{1}{d_{M}-d(t)} \int_{t-d_{M}}^{t-d(t)} x^{T}(\theta) d \theta\right] \\
& \times\left[\left(d_{M}-d(t)\right)\left(Z_{1}+\frac{1}{3} Z_{3}\right)\right] \\
& \times\left[x^{T}(t-d(t)), x^{T}\left(t-d_{M}\right), \frac{1}{d_{M}-d(t)} \int_{t-d_{M}}^{t-d(t)} x^{T}(\theta) d \theta\right]^{T} \\
& +\left(d_{M}-d_{m}\right) e^{-\alpha d_{M}}\left[x^{T}(t-d(t)), x^{T}\left(t-d_{M}\right), \frac{1}{d_{M}-d(t)} \int_{t-d_{M}}^{t-d(t)} x^{T}(\theta) d \theta\right] \\
& \times \operatorname{Sym}\left(N_{1}\left(x\left(t-d_{M}\right)-x(t-d(t))\right)\right) \\
& +\left(d_{M}-d_{m}\right) e^{-\alpha d_{M}}\left[x^{T}(t-d(t)), x^{T}\left(t-d_{M}\right), \frac{1}{d_{M}-d(t)} \int_{t-d_{M}}^{t-d(t)} x^{T}(\theta) d \theta\right] \\
& \times \operatorname{Sym}\left(N_{2}\left(\frac{2}{d_{M}-d(t)} \int_{t-d_{M}}^{t-d(t)} x^{T}(\theta) d \theta-x(t-d(t))-x\left(t-d_{M}\right)\right)\right) \\
& \leq-\alpha V_{3}+\left(d_{M}-d_{m}\right)^{2} \dot{x}^{T}(t) E^{T} X_{1} E \dot{x}(t) \\
& +\left(d_{M}-d_{m}\right) e^{-\alpha d_{M}}\left[x^{T}\left(t-d_{m}\right), x^{T}(t-d(t)), \frac{1}{d(t)-d_{m}} \int_{t-d(t)}^{t-d_{m}} x^{T}(\theta) d \theta\right] \\
& \times\left[\left(d_{M}-d_{m}\right)\left(Y_{1}+\frac{1}{3} Y_{3}\right)\right] \\
& \times\left[x^{T}\left(t-d_{m}\right), x^{T}(t-d(t)), \frac{1}{d(t)-d_{m}} \int_{t-d(t)}^{t-d_{m}} x^{T}(\theta) d \theta\right]^{T} \\
& +\left(d_{M}-d_{m}\right) e^{-\alpha d_{M}}\left[x^{T}\left(t-d_{m}\right), x^{T}(t-d(t)), \frac{1}{d(t)-d_{m}} \int_{t-d(t)}^{t-d_{m}} x^{T}(\theta) d \theta\right] \\
& \times \operatorname{Sym}\left(M_{1}\left(x(t-d(t))-x\left(t-d_{m}\right)\right)\right) \\
& +\left(d_{M}-d_{m}\right) e^{-\alpha d_{M}}\left[x^{T}\left(t-d_{m}\right), x^{T}(t-d(t)), \frac{1}{d(t)-d_{m}} \int_{t-d(t)}^{t-d_{m}} x^{T}(\theta) d \theta\right] \\
& \times \operatorname{Sym}\left(M_{2}\left(\frac{2}{d(t)-d_{m}} \int_{t-d(t)}^{t-d_{m}} x^{T}(\theta) d \theta-x(t-d(t))-x\left(t-d_{m}\right)\right)\right) \\
& +\left(d_{M}-d_{m}\right) e^{-\alpha d_{M}}\left[x^{T}(t-d(t)), x^{T}\left(t-d_{M}\right), \frac{1}{d_{M}-d(t)} \int_{t-d_{M}}^{t-d(t)} x^{T}(\theta) d \theta\right] \\
& \times\left[\left(d_{M}-d_{m}\right)\left(Z_{1}+\frac{1}{3} Z_{3}\right)\right]
\end{aligned}
$$




$$
\begin{aligned}
& \times\left[x^{T}(t-d(t)), x^{T}\left(t-d_{M}\right), \frac{1}{d_{M}-d(t)} \int_{t-d_{M}}^{t-d(t)} x^{T}(\theta) d \theta\right]^{T} \\
& +\left(d_{M}-d_{m}\right) e^{-\alpha d_{M}}\left[x^{T}(t-d(t)), x^{T}\left(t-d_{M}\right), \frac{1}{d_{M}-d(t)} \int_{t-d_{M}}^{t-d(t)} x^{T}(\theta) d \theta\right] \\
& \times \operatorname{Sym}\left(N_{1}\left(x\left(t-d_{M}\right)-x(t-d(t))\right)\right) \\
& +\left(d_{M}-d_{m}\right) e^{-\alpha d_{M}}\left[x^{T}(t-d(t)), x^{T}\left(t-d_{M}\right), \frac{1}{d_{M}-d(t)} \int_{t-d_{M}}^{t-d(t)} x^{T}(\theta) d \theta\right] \\
& \times \operatorname{Sym}\left(N_{2}\left(\frac{2}{d_{M}-d(t)} \int_{t-d_{M}}^{t-d(t)} x^{T}(\theta) d \theta-x(t-d(t))-x\left(t-d_{M}\right)\right)\right) .
\end{aligned}
$$

By applying the Jensen inequality in $\dot{V}_{4}\left(x_{t}\right)$, we have

$$
\begin{aligned}
\dot{V}_{4}\left(x_{t}\right) \leq & -\alpha V_{4}+d_{m}^{2} \dot{x}^{T}(t) E^{T} X_{2} E \dot{x}(t) \\
& -d_{m} e^{-\alpha d_{m}}\left(x(t)-x\left(t-d_{m}\right)\right)^{T} E^{T} X_{2} E\left(x(t)-x\left(t-d_{m}\right)\right) .
\end{aligned}
$$

Introducing the free weighting matrix $\mathcal{G}$, we have

$$
2 \xi^{T}(t) \mathcal{G}^{T}\left[-E \dot{x}_{i}(t)+A x(t)+D x(t-d(t))+B u(t)\right]=0,
$$

where

$$
\begin{aligned}
\xi(t)= & {\left[x^{T}(t), x^{T}\left(t-d_{m}\right), x^{T}(t-d(t)), x^{T}\left(t-d_{M}\right), \frac{1}{d(t)-d_{m}} \int_{t-d(t)}^{t-d_{m}} x^{T}(\theta) d \theta,\right.} \\
& \left.\frac{1}{d_{M}-d(t)} \int_{t-d_{M}}^{t-d(t)} x^{T}(\theta) d \theta,(E \dot{x}(t))^{T}, u^{T}(t)\right]^{T}, \\
\mathcal{G}= & {\left[G_{1}, G_{2}, G_{3}, G_{4}, G_{5}, G_{6}, G_{7}, G_{8}\right] . }
\end{aligned}
$$

Therefore, we have

$$
\begin{aligned}
\dot{V}= & \dot{V}_{\max }+\alpha V_{\max }\left(x_{t}\right)-\beta u^{T}(t) u(t) \\
& +2 \xi^{T}(t) \mathcal{G}^{T}\left[-E \dot{x}_{i}(t)+A x(t)+D x(t-d(t))+B u(t)\right] \\
\leq & \xi^{T}(t) \Omega \xi(t) .
\end{aligned}
$$

Therefore, let $\Delta=\dot{V}_{\max }+\alpha V_{\max }\left(x_{t}\right)-\beta u^{T}(t) u(t)$. Obviously, we have

$$
\Delta \leq \sum_{k=1}^{M} \gamma_{j k} x^{T}\left(p_{k}-p_{j}\right) x \leq 0
$$

According to Lemma 2.4, we have

$$
\|x(t, \phi)\| \leq \gamma_{2}+\left\{\left(\frac{\lambda_{2}}{\lambda_{1}}\|\phi\|^{2}-\frac{\beta u^{2}}{\alpha \lambda_{1}}\right)^{+}\right\}^{\frac{1}{2}} e^{-\alpha t}, \quad \forall t \geq 0,
$$


where

$$
\begin{aligned}
\gamma_{2}= & \sqrt{\frac{\beta u^{2}}{\alpha \lambda_{1}}}, \quad \lambda_{1}=\min _{1 \leq j \leq N} \lambda\left(E^{T} P_{j} E\right), \\
\lambda_{2}= & \max _{1 \leq j \leq N} \lambda\left(E^{T} P_{j} E\right)+\max _{1 \leq j \leq N} \lambda\left(Q_{1}\right) d_{m}+\max _{1 \leq j \leq N} \lambda\left(Q_{2}\right) d_{M}+\max _{1 \leq j \leq N} \lambda\left(Q_{3}\right) d_{M} \\
& +\max _{1 \leq j \leq N} \lambda\left(E^{T} X_{1} E\right)\left(d_{M}-d_{m}\right)^{3}+\max _{1 \leq j \leq N} \lambda\left(E^{T} X_{2} E\right) d_{m}^{2} .
\end{aligned}
$$

Remark 3 Define $\gamma_{1}=\frac{\lambda_{1}}{\lambda_{2}} \gamma_{2}$. It is obvious that $\lambda_{1}$ and $\lambda_{2}$ are positive constants due to the conditions from Theorem 1 . Denote $\mathcal{B}_{1}:=\mathcal{B}\left(\gamma_{1}\right), \mathcal{B}_{2}:=\mathcal{B}\left(\gamma_{2}\right)$, then $\mathcal{B}_{1} \subset \mathcal{B}_{2}$. According to the above inequality, it is easy for us to obtain two cases as regards the state trajectory of the singular system (1). On the one hand, for any initial condition which belongs to $\mathcal{B}_{1}$, the corresponding state trajectory is bounded within the ball $\mathcal{B}_{2}$. On the other hand, for any initial condition which is outside $\mathcal{B}_{1}$, as $t$ tends to infinity, the corresponding trajectory converges exponentially within the ball $\mathcal{B}_{2}$.

When taking $\phi(t)=0$, the singular system (1) is translated into the form

$$
\begin{aligned}
& E \dot{x}_{i}(t)=A x(t)+D x(t-d(t))+B u(t), \\
& x(t)=0 \quad t \in\left[-d_{M}, 0\right] .
\end{aligned}
$$

It is obvious that the new system (33) is just a special case of the system (1). The reachable set estimation for system (33) has been mainly studied by employing the common Lyapunov-Krasovskii functional in [17]. The corresponding bounding ellipsoids have also been derived. In this paper, we apply the maximal Lyapunov-Krasovskii functional to provide a non-ellipsoidal reachable set estimation for the system (1).

Define the reachable set of (1) as follows:

$$
\mathcal{R}_{x}=\left\{x(t) \in R^{n} \mid x(t), u(t) \text { satisfy }(1) \text { and } u^{T}(t) u(t) \leq u^{2}, t \geq 0\right\}
$$

From (34), it is easy to see that the reachable set of a system is regarded as a bounded set of all reachable states starting from the origin by input disturbances with constrained peak value. Since recently, the most interesting problem of estimation and control for dynamical systems has been to find an estimation of the bounds of the reachable sets. Therefore, many researchers have devoted efforts to investigating such conditions for deriving an ellipsoid or a non-ellipsoid which bounds the reachable set of the system.

Let $Q$ be a symmetric positive definite matrix and a scalar $r \geq 0$, the ellipsoid is defined as follows:

$$
\varepsilon(Q, r)=\left\{x \in R^{n}, x^{T} Q x \leq r\right\} .
$$

By employing the Lyapunov-Krasovskii functional in (19), from Lemma 2.4, we obtain

$$
V\left(x_{t}\right) \leq \frac{\beta u^{2}}{\alpha}\left(1-e^{-\alpha t}\right)<\frac{\beta u^{2}}{\alpha}, \quad \forall t \geq 0 .
$$

Note that $V\left(x_{t}\right) \geq x^{T}(t) E^{T} P_{j} E x(t)$. Therefore, we are ready to get $x^{T}(t) E^{T} P_{j} E x(t) \leq \frac{\beta u^{2}}{\alpha}$. By combining the definitions about $\mathcal{R}_{x}, \varepsilon(Q, r)$, it is not difficult to get the reachable set $\mathcal{R}_{x}$ 
of system (1) under zero initial condition. The reachable set is bounded by the ellipsoid $\varepsilon\left(E^{T} P_{j} E, r^{*}\right)$, where $r^{*}=\frac{\beta u^{2}}{\alpha}$.

$x^{T}(t) E^{T} P_{j} E x(t) \leq \frac{\beta u^{2}}{\alpha}$ stands for $\hat{x}^{T}(t) T^{T} E^{T} S^{T} S^{-T} P_{j} S^{-1} S E T \hat{x}(t) \leq \frac{\beta u^{2}}{\alpha}$, that is to say, $\hat{x}^{T}(t) \hat{P}_{j} \hat{x}(t) \leq \frac{\beta u^{2}}{\alpha}$ with $\hat{P}_{j}=\left(S^{-T} P_{j} S^{-1}\right)_{n_{1} \times n_{1}}$.

Thus, the following inequality holds:

$$
\left\|\hat{x}_{1}(t)\right\| \leq \epsilon_{1}
$$

with $\epsilon_{1}=\frac{\beta u^{2}}{\alpha \sqrt{\min _{1 \leq j \leq N} \lambda\left(\hat{P}_{j}\right)}}$. Obviously, $\left\|\hat{x}_{1}(t)\right\|$ is bounded.

On the other hand, from Assumption 1, it follows from Lemma 2.3 that

$$
\begin{aligned}
\left\|\hat{x}_{2}(t)\right\| & =\left\|\hat{D}_{21} \hat{x}_{1}(t-\tau(t))+\hat{D}_{22} \hat{x}_{2}(t-\tau(t))+\hat{B}_{2} u(t)\right\| \\
& \leq\left\|\hat{D}_{21}\right\| \epsilon_{1}+\left\|\hat{D}_{22}\right\|\left\|\hat{x}_{2}(t-\tau(t))\right\|+\left\|\hat{B}_{2}\right\| u .
\end{aligned}
$$

By employing Lemma 6 in [17], $\left\|\hat{x}_{2}(t)\right\| \leq \epsilon_{2}$. That is, $\hat{x}_{2}^{T}(t) \frac{1}{\epsilon_{2}^{2}} \hat{x}_{2}(t) \leq 1$.

It yields $\left\|\hat{x}_{2}(t)\right\| \leq \sup _{-d_{M} \leq t \leq 0}\|x(t)\|+\frac{\left\|\hat{D}_{21}\right\| \epsilon_{1}+\left\|\hat{B}_{2}\right\| u}{1-\left\|\hat{D}_{22}\right\|}=\|\phi\|+\frac{\left\|\hat{D}_{21}\right\| \epsilon_{1}+\left\|\hat{B}_{2}\right\| u}{1-\left\|\hat{D}_{22}\right\|}$. We have

$$
\hat{x}_{2}^{T}(t) \frac{1}{\epsilon_{2}^{2}} \hat{x}_{2}(t) \leq 1
$$

with $\epsilon_{2}=\|\phi\|+\frac{\left\|\hat{D}_{21}\right\| \epsilon_{1}+\left\|\hat{B}_{2}\right\| u}{1-\left\|\hat{D}_{22}\right\|}$.

Then adding the inequality in (37) times $\eta$ and the inequality in (39) times $(1-\eta) \frac{\beta u^{2}}{\alpha}$, we obtain

$$
\left[\begin{array}{l}
\hat{x}_{1}(t) \\
\hat{x}_{2}(t)
\end{array}\right]^{T}\left[\begin{array}{cc}
\eta \hat{P}_{j} & 0 \\
0 & \frac{(1-\eta) \beta u^{2}}{\epsilon_{2}^{2} \alpha}
\end{array}\right]\left[\begin{array}{l}
\hat{x}_{1}(t) \\
\hat{x}_{2}(t)
\end{array}\right] \leq \frac{\beta u^{2}}{\alpha} .
$$

That is,

$$
x^{T} \hat{\hat{P}}_{j} x \leq \frac{\beta u^{2}}{\alpha}, \quad \hat{P}_{j}=\left(S^{-T} P_{j} S^{-1}\right)_{n_{1} \times n_{1}}, \quad \hat{\hat{P}}_{j}=T^{-T}\left[\begin{array}{cc}
\eta \hat{P}_{j} & 0 \\
0 & \frac{(1-\eta) \beta u^{2}}{\epsilon_{2}^{2} \alpha}
\end{array}\right] T^{-1} .
$$

The next thing is to certify the regularity and non-impulsiveness characteristics of system (1). It is not easy to get

$$
\begin{aligned}
& \tilde{E}^{T} \tilde{P}_{j}=\tilde{P}_{j}^{T} \tilde{E}=\left[\begin{array}{cc}
E^{T} P_{j} E & 0 \\
0 & 0
\end{array}\right] \geq 0, \\
& \operatorname{sym}\left(\tilde{A}^{T} \tilde{P}_{j}\right)+\tilde{Q}-\tilde{E}^{T} \tilde{X} \tilde{E}=\left[\begin{array}{cc}
\Omega_{11} & \Omega_{17} \\
* & \Omega_{77}
\end{array}\right]<0,
\end{aligned}
$$

where

$$
\tilde{A}=\left[\begin{array}{cc}
0 & I \\
A & -I
\end{array}\right], \quad \tilde{E}=\left[\begin{array}{cc}
E & 0 \\
0 & 0
\end{array}\right],
$$




$$
\begin{aligned}
& \tilde{X}=\left[\begin{array}{cc}
e^{-\alpha d_{m}} X_{2} & 0 \\
0 & 0
\end{array}\right], \quad \tilde{P}_{j}=\left[\begin{array}{cc}
P_{j} E+E_{0} U & 0 \\
G_{1} & G_{7}
\end{array}\right], \\
& \tilde{Q}=\left[\begin{array}{cc}
Q_{1}+Q_{2}+Q_{3}+\alpha E^{T} P_{j} E & 0 \\
0 & d_{m}^{2} X_{2}+\left(d_{M}-d_{m}\right)^{2} X_{1}
\end{array}\right] .
\end{aligned}
$$

From (41)-(42), we get

$$
\operatorname{sym}\left(\tilde{A}^{T} \tilde{P}_{j}\right)-\tilde{E}^{T} \tilde{Z} \tilde{E}<0 .
$$

Because $\operatorname{rank}(\tilde{E})=\operatorname{rank}(E)=n_{1} \leq n$, there exist nonsingular matrices $\tilde{S}$ and $\tilde{T}$ such that

$$
\bar{E}=\tilde{S} \tilde{E} \tilde{T}=\left[\begin{array}{ll}
I & 0 \\
0 & 0
\end{array}\right]
$$

Denote

$$
\bar{A}=\tilde{S} \tilde{A} \tilde{T}=\left[\begin{array}{ll}
A_{11} & A_{12} \\
A_{21} & A_{22}
\end{array}\right], \quad \bar{P}=\tilde{S}^{-T} \tilde{P} \tilde{N}=\left[\begin{array}{ll}
P_{11} & P_{12} \\
P_{21} & P_{22}
\end{array}\right] .
$$

Taking (43) into account, we have $P_{12}=0$ and $P_{11}>0$. Then pre-multiplying and postmultiplying (43) by $\tilde{T}^{T}$ and $T$, respectively, it is easy to obtain $\operatorname{sym}\left(A_{22}^{T} P_{22}\right)<0$ showing that $A_{22}$ is nonsingular. Thus, the pair $(\tilde{E}, \tilde{A})$ is regular and impulse free. In addition, by simple computation, it is easy to certify that $\operatorname{det}(s E-A)=\operatorname{det}(s \tilde{E}-\tilde{A})$ and $\operatorname{deg}(\operatorname{det}(s E-A))=$ $\operatorname{deg}(\operatorname{det}(s \tilde{E}-\tilde{A}))$. Finally, it is obvious that the system (1) is regular and impulse free.

\section{Numerical examples}

In this section, a numerical simulation example will be presented to demonstrate the effectiveness of our obtained results.

Example 1 Consider the two dimensional system (1) with

$$
\begin{aligned}
& E=\left[\begin{array}{c}
-0.6 \\
1.1
\end{array}\right], \quad A=\left[\begin{array}{cc}
-2 & 0 \\
0 & -0.7
\end{array}\right], \quad B=\left[\begin{array}{cc}
-1 & 0 \\
-1 & -1
\end{array}\right], \\
& u(t)=\left[\begin{array}{c}
\cos (t) \\
\sin (t)
\end{array}\right], \quad D=0 .
\end{aligned}
$$

We have given

$$
P_{1}=\left[\begin{array}{ll}
0.4597 & 0.0270 \\
0.0270 & 0.3100
\end{array}\right], \quad P_{2}=\left[\begin{array}{cc}
0.8599 & 0.7400 \\
-0.7400 & 2.1083
\end{array}\right] \text {. }
$$

Figures 1 and 2 describe the reachable set estimation for the singular system (1) with $\phi(t)=0$. 


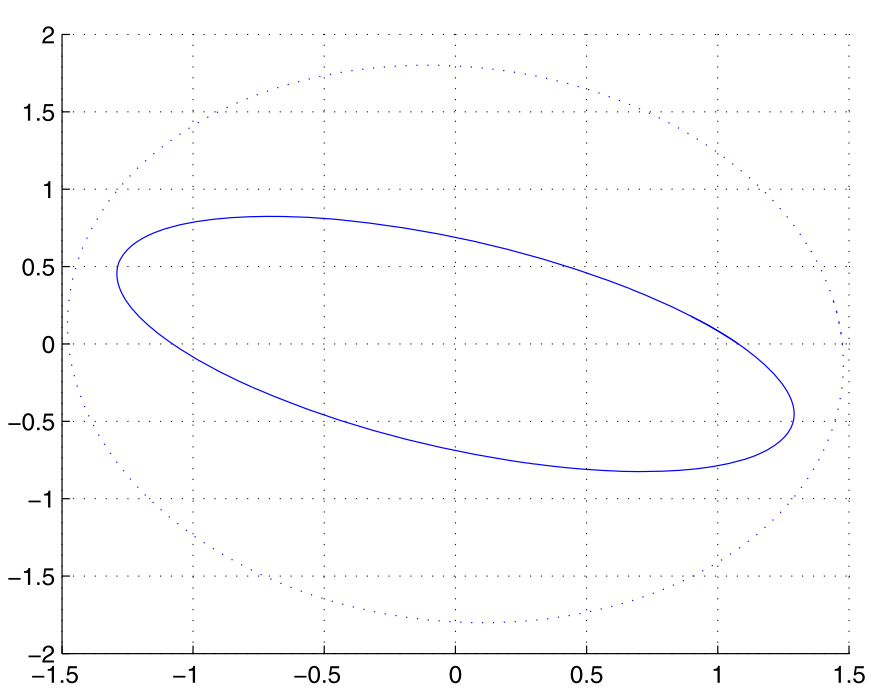

Figure 1 The reachable set estimation for the singular system (1) with $\phi(t)=0$

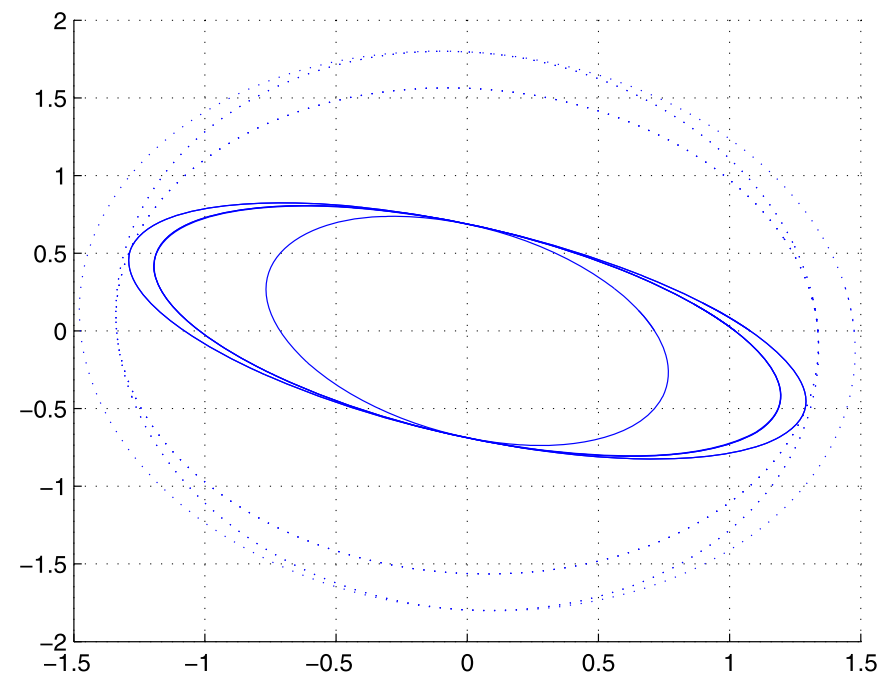

Figure 2 The reachable set estimation for the singular system (1) with $\phi(t)=0$

\section{Conclusions}

The problem of a state bounding estimation of a continuous-time singular system with time delays has been investigated in this paper. Some proper conditions have been established to guarantee the state bounding set for the singular system with time delays by using the maximal Lyapunov-Krasovskii functional and employing the new free-matrix-based integral inequality. The above methods can be extended to our future studies, such as of fractional-order systems and memristor-based neural networks. 
Funding

This work was supported by Young scholars development fund of swpu (No. 200331010060).

\section{Competing interests}

The authors declare that they have no competing interests.

\section{Authors' contributions}

All authors contributed equally to the writing of this paper. All authors read and approved the manuscript.

\section{Publisher's Note}

Springer Nature remains neutral with regard to jurisdictional claims in published maps and institutional affiliations.

Received: 10 May 2018 Accepted: 14 March 2019 Published online: 22 March 2019

\section{References}

1. Liu, G., Xu, S., Wei, Y., Qi, Z., Zhang, Z.: New insight into reachable set estimation for uncertain singular time-delay systems. Appl. Math. Comput. 320, 769-780 (2018)

2. Liu, G., Xu, S., Park, J.H., Zhang, G.: Reliable exponential H filtering for singular Markovian jump systems with time-varying delays and sensor failures. Int. J. Robust Nonlinear Control 28, 4230-4245 (2018)

3. Liu, G., Wei, Y., Ma, Q., Lu, J., Chu, Y.: Robust non-fragile guaranteed cost control for singular Markovian jump time-delay systems. Trans. Inst. Meas. Control 40, 2141-2150 (2018)

4. Liu, G., Ma, Q., Lu, J.: Mixed H-infinity and passive filtering for a class of singular systems with interval time-varying delays. Optim. Control Appl. Methods 39, 377-392 (2018)

5. Liu, G., Qi, Z., Xu, S.: alpha-dissipativity filtering for singular Markovian jump systems with distributed delays. Signal Process. 134, 149-157 (2017)

6. Fridman, E., Pila, A., Shaked, U.: Regional stabilization and $\mathrm{H}$ control of time-delay systems with saturating actuators. Int. J. Robust Nonlinear Control 13, 885-907 (2003)

7. Fridman, E., Shaked, U.: On reachable sets for linear systems with delay and bounded peak inputs. Automatica 39 , 2005-2010 (2003)

8. He, Y., Wang, Q., Lin, C., Wu, M.: Delay-range-dependent stability for systems with time-varying delay. Automatica 43, 371-376 (2007)

9. Park, J.H., Park, C.H., Kwon, O.M., Lee, S.M.: A new stability criterion for bidirectional associative memory neural networks of neutral-type. Appl. Math. Comput. 199, 716-722 (2008)

10. Fridman, E., Shaked, U., Liu, K.: New conditions for delay-derivative-dependent stability. Automatica 45, 2723-2727 (2009)

11. Kim, J.H.: Improved ellipsoidal bound of reachable sets for time-delayed linear systems with disturbances. Automatica 44, 2940-2943 (2008)

12. Kwon, O., Lee, S., Park, J.: On the reachable set bounding of uncertain dynamic systems with time-varying delays and disturbances. Inf. Sci. 181, 3735-3748 (2011)

13. Nam, P., Pathirana, P.: Further result on reachable set bounding for linear uncertain polytopic systems with interval time-varying delays. Automatica 47, 1838-1841 (2011)

14. Zuo, Z., Ho, D.W.C., Wang, Y.: Fault tolerant control for singular systems with actuator saturation and nonlinear perturbation. Automatica 46, 569-576 (2010)

15. Zuo, Z., Ho, D.W.C., Wang, Y.: Reachable set bounding for delayed systems with polytopic uncertainties: the maximal Lyapunov-Krasovskii functional approach. Automatica 46, 949-952 (2010)

16. Oucheriah, S.: Robust exponential convergence of a class of linear delayed systems with bounded controllers and disturbances. Automatica 42, 1863-1867 (2006)

17. Feng, Z.G., Lam, J.: On reachable set estimation of singular systems. Automatica 52, 146-153 (2015)

18. Zeng, H.B., He, Y., Wu, M., She, J.H.: Free-matrix-based integral inequality for stability analysis of systems with time-varying delay. IEEE Trans. Autom. Control 60, 2768-2772 (2015)

19. de Oliveira, M.C., Skelton, R.E.: Stability tests for constrained linear systems. In: Perspectives in Robust Control, pp. 241-257. Springer, Berlin (2001)

20. Xiao, J.Y., Zhong, S.M.: Extended dissipative conditions for memristive neural networks with multiple time delays. Appl. Math. Comput. 323, 145-163 (2018)

21. Elsgolts, L.E., Norkin, S.B.: Introduction to the Theory of the Differential Equations with Deviating Argument. Academic Press, New York (1973)

22. Khusainov, D.Ya., Shatyrko, A.V.: Absolute stability of multi-delay regulation systems. J. Autom. Inf. Sci. 27(3-4), 33-42 (1995)

23. Shatyrko, A.V., Khusainov, D.Ya.: Investigation of absolute stability of nonlinear systems of special kind with aftereffect by Lyapunov functions method. J. Autom. Inf. Sci. 43(7), 61-75 (2011)

24. Shatyrko, A., Khusainov, D.: On the Interval Stability of Weak-Nonlinear Control Systems with Aftereffect. Sci. World J. https://doi.org/10.1155/2016/6490826

25. Zeng, D., Zhang, R., Liu, Y., Zhong, S.: Sampled-data synchronization of chaotic Lur'e systems via input-delay-dependent-free-matrix zero equality approach. Appl. Math. Comput. 315, 34-46 (2017)

26. Zhang, R., Zeng, D., Zhong, S., Shi, K., Cui, J.: New approach on designing stochastic sampled-data controller for exponential synchronization of chaotic Lur'e systems. Nonlinear Anal. Hybrid Syst. 29, 303-321 (2018)

27. Wang, X., Liu, X., She, K., Zhong, S.: Pinning impulsive synchronization of complex dynamical networks with various time-varying delay sizes. Nonlinear Anal. Hybrid Syst. 26, 307-318 (2017)

28. Wang, X., Liu, X., She, K., Zhong, S., Shi, L.: Delay-dependent impulsive distributed synchronization of stochastic complex dynamical networks with time-varying delays. IEEE Trans. Syst. Man Cybern. (2018). https://doi.org/10.1109/tsmc.2018.2812895 
29. Shan, Y., Zhong, S., Cui, J., Hou, L., Li, Y.: Improved criteria of delay-dependent stability for discrete-time neural networks with leakage delay. Neurocomputing 266, 409-419 (2017)

30. Shan, Y., She, K., Zhong, S., Zhong, Q., Shi, K., Zhao, C.: Exponential stability and extended dissipativity criteria for generalized discrete-time neural networks with additive time-varying delays. Appl. Math. Comput. 333, 145-168 (2018)

31. Chen, H., Zhang, Z., Wang, H.: Robust H state-feedback control for linear system. Proc. R. Soc. A 473, 20160934 (2017)

32. Chen, H., Zhong, S., Liu, X., Li, Y., Shi, K.: Improved results on nonlinear perturbed T-S fuzzy system with mixed delays. J. Franklin Inst. 354, 2032-2052 (2017)

33. Xie, W., Zhu, H., Zhong, S., Zhang, D., Shi, K., Cheng, J.: Extended dissipative estimator design for uncertain switched delayed neural networks via a novel triple integral inequality. Appl. Math. Comput. 335, 82-102 (2018)

34. Xie, W., Zhu, H., Zhong, S., Cheng, J., Shi, K.: Extended dissipative resilient estimator design for discrete-time switched neural networks with unreliable links. Nonlinear Anal. Hybrid Syst. 32, 19-36 (2019)

35. Cheng, J.: Ahn, C.K., Karimi, H.R., Cao, J., Qi, W.: An event-based asynchronous approach to Markov jump systems with hidden mode detections and missing measurements. IEEE Trans. Syst. Man Cybern. Syst. (2018). https://doi.org/10.1109/TSMC.2018.2866906

36. Cheng, J., Chang, X.H., Park, J.H., Li, H., Wang, H.: Fuzzy-model-based $H_{\infty}$ control for discrete-time switched systems with quantized feedback and unreliable links. Inf. Sci. 436-437, 181-196 (2018)

37. Wang, B., Zhang, D., Cheng, J., Park, J.H.: Fuzzy model-based nonfragile control of switched discrete-time systems. Nonlinear Dyn. 93(4), 2461-2471 (2018)

38. Shi, K., Tang, Y., Zhong, S., Yin, C., Huang, X., Wang, W.: Nonfragile asynchronous control for uncertain chaotic Lurie network systems with Bernoulli stochastic process. Int. J. Robust Nonlinear Control 28(5), 1693-1714 (2018)

39. Wang, J., Shi, K., Huang, Q., Zhong, S., Zhang, D.: Stochastic switched sampled-data control for synchronization of delayed chaotic neural networks with packet dropout. Appl. Math. Comput. 335, 211-230 (2018)

40. Shi, K., Wang, J., Zhong, S., Zhang, X., Zhong, S., Liu, Y., Cheng, J.: New reliable nonuniform sampling control for uncertain chaotic neural networks under Markov switching topologies. Appl. Math. Comput. 347, 169-193 (2019)

41. Xiao, J., Zhong, S., Li, Y., Xu, F.: Relaxed exponential passivity criteria for memristor-based neural networks with leakage and time-varying delays. Int. J. Mach. Learn. Cybern. 8, 1875-1886 (2017)

42. Xiao, J., Zhong, S., Xu, F.: Design disturbance attenuating controller for memristive recurrent neural networks with mixed time-varying delays. Adv. Differ. Equ. (2018). https://doi.org/10.1186/s13662-018-1641-8

43. Yang, H., Wang, X., Zhong, S., Shu, L.: Synchronization of nonlinear complex dynamical systems via delayed impulsive distributed control. Appl. Math. Comput. 320, 75-85 (2018)

44. Wang, X., She, K., Zhong, S.: Finite-time lag synchronization of master-slave complex dynamical networks with unknown signal propagation delays. J. Franklin Inst. 354(12), 4913-4929 (2017)

45. Zhang, R., Zeng, D., Park, J.H., Liu, Y., Zhong, S.: Quantized sampled-data control for synchronization of inertial neural networks with heterogeneous time-varying delays. IEEE Trans. Neural Netw. Learn. Syst. 29(12), 6385-6395 (2018)

46. Xiao, J., Li, Y., Zhong, S., Xu, F.: Extended dissipative state estimation for memristive neural networks with time-varying delay. ISA Trans. 64, 113-128 (2016)

47. Xiao, J.Y., Zhong, S.M., Li, Y.T., Xu, F.: Finite-time Mittag-Leffler synchronization of fractional-order memristive BAM neural networks with time delays. Neurocomputing 219, 431-439 (2017)

48. Zhang, R. Liu, X., Zeng, D., Zhong, S., Shi, K.: A novel approach to stability and stabilization of fuzzy sampled-data Markovian chaotic systems. Fuzzy Sets Syst. 344, 108-128 (2018)

49. Zhang, R., Zeng, D., Zhong, S.: Novel master-slave synchronization criteria of chaotic Lur'e systems with time delays using sampled-data control. J. Franklin Inst. 354(12), 4930-4954 (2017)

50. Xiao, J.Y., Zhong, S.M., Li, Y.T.: New passivity criteria for memristive uncertain neural networks with leakage and time-varying delays. ISA Trans. 59, 133-148 (2015)

51. Xiao, J.Y., Zhong, S.M., Li, Y.T.: Relaxed dissipativity criteria for memristive neural networks with leakage and time-varying delays. Neurocomputing 171,707-718 (2016)

52. Xiao, J.Y., Zhong, S.M., Li, Y.T.: Improved passivity criteria for memristive neural networks with interval multiple time-varying delays. Neurocomputing 171, 1414-1430 (2016)

53. Shi, Y., Cao, J.D., Chen, G.R.: Exponential stability of complex-valued memristor-based neural networks with time-varying delays. Appl. Math. Comput. 313, 222-234 (2017)

\section{Submit your manuscript to a SpringerOpen ${ }^{\circ}$ journal and benefit from:}

- Convenient online submission

- Rigorous peer review

- Open access: articles freely available online

- High visibility within the field

- Retaining the copyright to your article

Submit your next manuscript at $\boldsymbol{~ s p r i n g e r o p e n . c o m ~}$ 